\title{
Economic Status and Maternal Health: Analysis of The 2013-14 Zambia Demographic and Health Survey
}

\author{
Mulenga Chonzi Mulenga \\ Jawaharlal Nehru University, India
}

Corresponding Author: Mulenga Chonzi Mulenga: mulengachonzi2002@gmail.com

\section{Citation: Mulenga M. C. (2017) Economic Status and Maternal Health: Analysis of The 2013-14 Zambia Demographic and Health Survey. Open Science Journal 2(2).}

Received: $11^{\text {th }}$ January 2017

Accepted: 20 ${ }^{\text {th }}$ March 2017

Published: $4^{\text {th }}$ May 2017

Copyright:@ 2016 This is an open access article under the terms of the Creative Commons

Attribution License, which permits unrestricted use, distribution, and reproduction in any medium, provided the original author and source are credited.

\section{Funding: The author(s) received} no specific funding for this work.

\section{Competing Interests: The} author have declared that no competing interests exists.

\section{Abstract}

With the current high maternal mortality rates prevailing in Zambia, it is imperative to analyze the effect that the economic status of women has on maternal health outcomes. It is hypothesized that wealth is positively linked to better health outcomes. As a result, maternal health outcomes will improve when the economic status of women improve. Using data for women from the 2013-14 Zambia Demographic and Health Survey, bivariate and multivariate analysis was used to ascertain the impact of wealth as a proxy for economic status and other variables based on five independent outcomes (Number of antenatal care visits, timing of first antenatal care visit, tetanus toxoid vaccination, iron tablet/syrup uptake, and place of delivery) and a composite measure of maternal health. The results revealed that women's wealth and region were the only significant factor associated with all the five independent indicators and the composite measure of maternal health. However, for the composite measure, literacy and age of the mother were also found to be associated with maternal health. The study therefore concluded that economic status is an important factor in improving maternal health outcomes in Zambia. As a result, policy should be aimed at providing support to women within the lowest wealth quintiles so as to enable them access maternal health services. 
Keywords: Economic Status, Wealth Index, Wealth, Maternal Health.

\section{Introduction}

Every day, women die from pregnancy or childbirth-related complications around the world, and this is particularly rife in developing countries. Statistics show that in 2015, out of 303000 global maternal deaths, 99\% (302 000) occurred in the developing region with Sub-Saharan Africa (SSA) alone accounting for roughly $66 \%$ (201 000) [1]. According to a report by the [2], the causes of maternal deaths can be categorized into two: Direct and Indirect. The direct causes of maternal mortality in Zambia are post-partum hemorrhage (34\%), sepsis (13\%), obstructed labor (8\%), pregnancy hypertensive disorders eclampsia (5\%), and abortion complications (4\%) while indirect causes include malaria (11\%), HIV (10\%), and others (17\%) [2].

These deaths are however preventable with quality maternal health care throughout pregnancy and childbirth as many studies have shown $[3,4,5,6]$. According to [7], all women need access to antenatal care in pregnancy, skilled care during childbirth, and care and support in the weeks after childbirth; and in addition, it is also vital to prevent unwanted and too-early pregnancies. The Ministry of Health and the World Health Organization recommends that women seek antenatal care, preferably at 14 weeks; consequently, they should make a minimum of four antenatal visits before they give birth [5]. In addition, each visit should be conducted by a skilled health provider, preferably a midwife or doctor [5]. The Ministry of Health also recommends that women should give birth in a health centre or hospital where they can access skilled attendance from midwives and doctors; and after giving birth, women are advised to return to the health facility for postnatal care, preferably within the first 6 days postpartum [5]. All these are aspects of maternal health and are seen as important elements in reducing maternal morbidity and mortality. Maternal health according to the [8] refers to the health of women during pregnancy, childbirth, and the postpartum period. Thus, it has a close relationship with the right to the highest attainable standard of health [9].

Maternal health in Zambia - a SSA country remains unfavorable despite significant strides being made to reduce maternal deaths. According to the 201314 Zambia Demographic and Health Survey (ZDHS), maternal deaths represent 9.5\% (151) of all deaths among women aged 15-49 [10]. In order to reduce maternal deaths and ensure optimal maternal health outcomes, the Zambian Government with its collaborating partners has come up with many initiatives which are in line with WHO guidelines. Some of the initiatives include: The Zambia Safe Motherhood Initiative (SMI) launched in 1987 by the Government of Zambia with the support of the United Nations International Children's Emergency Fund (UNICEF) in an effort to halve the number of maternal deaths by the year 2000 [11]. The Maternal and Neonatal Health (MNH) project, supported by the United States Agency for International Development (USAID), was set up in 2000 to strengthen essential maternal and neonatal services using improved delivery of service, behavioral change, and Policy \& Advocacy 
interventions [11]. The Road Map for Accelerating the Reduction of Maternal and Neonatal Mortality launched in 2007 emphasized Governments priorities in achieving Millennium Development Goal's 4 \& 5 by 2015 [12]. The Global Health Initiative (GHI) 2011 - 2015 was initiated by the United States to directly support the Government's national health and development goals to reduce maternal, neonatal, and childhood deaths [12].

\section{Problem Statement}

Despite all the initiatives being put in place by the Government and its cooperating partners, maternal deaths still remain rampant in Zambia. The country continues to face high levels of maternal mortality with an average of 38 women dying every month due to pregnancy and childbirth [13] and close to 500 women out of every 100,000 dying in the process of giving birth [14]. This study therefore tries to explore the effect of economic status on maternal health care service utilization. According to [15], economic status is signified by the wealth of the individual/family. The hypothesis made here states that wealth is positively linked to better health outcomes. Therefore, it is expected that maternal health improves when the economic status of women improve. This study is unique in the sense that it uses an index of wealth to measure economic status, while several studies conducted in low-income countries have used socioeconomic status as an important determinant of maternal health care service utilization $[4,16,17$, 18]. The wealth index is used because it is a much broader indicator of wealth as it is consistent with expenditure and income measures [20]. This study also adds to literature in Zambia as similar studies undertaken in Zambia [5, 19] have not explored the effect of economic status on maternal health care. Empirical studies undertaken in other developing countries have shown that there are several other factors that affect maternal health care. These factors can, however, be classified into two: Socio-economic and demographic. Socio-economic factors include region, place of residence, wealth index, literacy and Access to Media, while Demographic factors include Mothers age at first child birth and Marital Status [15, 20, 21]. Also, this study uses a composite measure of five different variables (number of antenatal care (ANC) visits, timing of first ANC visit, tetanus toxoid (TT) vaccination, iron tablet/syrup uptake, and place of delivery) as a proxy for maternal health services. This is a refinement to studies undertaken by [15] in Ghana who only used ANC as a proxy for maternal health service utilization; [19] who only examined two aspects of maternal health care: ANC and facility based deliveries; and [22] who used four indicators: making at least one antenatal care visit; making four or more antenatal care visits; initiating antenatal care within the first trimester; and giving birth in a health facility.

\section{Research Questions}

1) Does the economic status of the mother affect maternal health in Zambia?

2) What other factors are associated with maternal health in Zambia? 


\section{Materials and Methods}

This section presents information used by the study on the extent to which women in Zambia receive care during pregnancy and delivery as obtained by the 2013-14 Zambia Demographic and Health Survey (ZDHS).

\section{Data used in the Analysis}

This study used data for women from the 2013-14 ZDHS to assess the impact of economic status on maternal health. The ZDHS is a key source of nationally representative data on population and health indicators, including maternal health. The study adopted the ZDHS complex two-stage stratified cluster sample design with Enumeration Areas (or clusters) selected during the first stage and households selected during the second stage [10]. The study involved all women of reproductive age (15-49) who reported a live birth in the five years preceding the survey. In addition, data was also restricted to the most recent birth during that period.

\section{Definition of Variables}

\section{Dependent Variable}

The dependent variable used in the study was maternal health care services utilization as a measure of maternal health. This variable choice is based on the hypothesis that the higher the use of maternal health care services, the better the maternal health outcomes. The study explores five outcomes of the use of maternal health care services for the most recent birth: Number of antenatal care visits, timing of first antenatal care visit, tetanus toxoid vaccination, iron tablet/syrup taken, and place of delivery.

All the 5 independent indicators for the dependent variable were dichotomous: Number of times of antenatal care (anc4) visits was measured based on whether the number of ANC visits the mother made for the last birth was four or more times or not. For the timing of the visit (anc3), the study constructed a measure of whether the woman made her first antenatal care visit during or after the first three months of her pregnancy based on the question on the timing of the first antenatal care visit. The measure was based on the [23] recommendation that in order to detect and effectively treat underlying problems, the first antenatal care visit should occur as early as possible, and preferably within the first trimester; For receipt of tetanus taxoid vaccine (tetanus_taxoid), whether or not mothers received a tetanus toxoid vaccine; For receipt of iron tablets/syrup (h42_1), whether or not mother received iron tablets/syrup; For place of delivery $\left(\mathrm{m} 15_{-} 1\right)$, whether or not the birth was delivered in a health facility or at home.

The study also constructed a composite measure of maternal health (maternal_health) incorporating all the 5 independent indicators as a separate outcome variable. The composite measure was a dichotomous variable which was coded $(0=$ Poor Maternal Health; and $1=$ Good Maternal Health). Multivariate binary logistic regression was used in both stages. 


\section{Independent Variables}

The main independent variable in the model was economic status which was measured by the wealth index (v190). The index was constructed using household asset data via a principal components analysis [10]. The DHS wealth quintiles were grouped into a five-category wealth status variable coded as poorest, poorer, middle, richer, and richest. Other social, economic and demographic factors included region (v024) for the 10 provinces in Zambia; place of residence (v025), a dichotomous variable which was coded $(1=$ urban and $2=$ rural); literacy (v155) recoded as a dichotomous variable $(0=$ not literate and $1=$ literate) was used as a proxy for education; access to media (media_exposure) was constructed as a combination of three variables (frequency of reading newspapers or magazines, listening to radio, and watching television) and recoded as a dichotomous variable $(0=$ no access/exposure and $1=$ access to media/ exposed); mothers age at first child birth (age_birth) coded into three categories $(1=<20,2=20-34$ and $3=35+$ ); and marital status (marital_status) was also recoded as a dichotomous measure $(0=$ never married and $1=$ married). Subsequently, it may be important to state that each of the independent variables was selected for inclusion in the analysis based on previous literature on similar studies undertaken in other Least Developing Countries.

\section{Statistical Analysis}

Bivariate and Multivariate analysis was conducted in the study. Bivariate analysis was used to describe the different maternal health variables by wealth quintile, while multivariate analysis involved two stages. The first stage involved constructing five models for each of the 5 indicators of maternal health and then running regressions for each indicator in order to identify their individual determinants. The second stage involved constructing a composite measure for maternal health using all the 5 indicators in order to ascertain the impact of economic status and other variables on maternal health.

\section{Results}

Table 1 shows the percentage distribution of the 5 maternal health indicators used in the study according to wealth quintile for the most recent birth. The results reveal that $53 \%$ of the women interviewed made 4 or more ANC visits of which the majority were in the richest wealth quintile $(65 \%)$. The results also revealed that out of the $47 \%$ that made less than 4 ANC visits, the women in the poorest wealth category had the highest proportion (52\%) compared to the women in the richest wealth group $(35 \%)$. 
Table 1. Percentage Distribution of Maternal Health Indicators according to Wealth Quintile

\begin{tabular}{|c|c|c|c|c|c|c|c|c|c|}
\hline & & & \multicolumn{7}{|c|}{ Wealth Quintile } \\
\hline & & & poorest & poorer & middle & richer & richest & Total & p-value \\
\hline & $<4$ & $\%$ & 51.6 & 46.9 & 47.1 & 48.5 & 34.7 & 46.6 & \\
\hline & $<4$ & $\mathrm{CI}$ & {$[48.5,54.7]$} & $43.2,50.6$ & {$[43.2,51.0]$} & {$[43.3,53.8]$} & {$[30.1,39.6]$} & {$[44.7,48.7]$} & \\
\hline \multirow[t]{6}{*}{ had 4 or more ANC visits } & $4+$ & $\%$ & 48.4 & 53.1 & 52.9 & 51.5 & 65.3 & 53.4 & $0.000^{* * *}$ \\
\hline & $4+$ & $\mathrm{CI}$ & {$[45.3,51.5]$} & {$[49.4,56.8]$} & {$[49.0,56.8]$} & {$[46.2,56.7$} & {$[60.4,69.9]$} & {$[51.3,55.3]$} & \\
\hline & Total & $\%$ & 100 & 100 & 100 & 100 & 100 & 100 & \\
\hline & $\mathrm{N}$ & & 1,236 & 1,149 & 1,009 & 881 & 713 & 4,989 & \\
\hline & $>3+$ & $\%$ & 74.5 & 77 & 78.4 & 78.7 & 69.6 & 75.9 & \\
\hline & $>3+$ & $\mathrm{CI}$ & {$[71.0,77.7]$} & {$[73.9,79.8]$} & {$[74.8,81.5]$} & {$[74.6,82.2]$} & {$[64.8,74.0]$} & {$[74.1,77.6]$} & \\
\hline First antenatal within 3 & 3 & $\%$ & 25.5 & 23 & 21.6 & 21.3 & 30.4 & 24.1 & $0.007^{* *}$ \\
\hline \multirow{5}{*}{ months } & 3 & $\mathrm{CI}$ & {$[22.3,29.0]$} & {$[20.2,26.1]$} & {$[18.5,25.2]$} & {$[17.8,25.4]$} & {$[26.0,35.2]$} & {$[22.4,25.9]$} & \\
\hline & Total & $\%$ & 100 & 100 & 100 & 100 & 100 & 100 & \\
\hline & $\mathrm{N}$ & & 1,205 & 1,130 & 998 & 872 & 712 & 4,918 & \\
\hline & no & $\%$ & 25.1 & 25.2 & 23.4 & 15.1 & 14.1 & 21.4 & \\
\hline & no & CI & {$[22.2,28.1]$} & {$[22.5,28.1]$} & {$[20.4,26.8]$} & {$[12.2,18.4]$} & {$[11.0,17.9]$} & {$[20.0,22.9]$} & \\
\hline \multirow{4}{*}{$\begin{array}{l}\text { received tetanus taxoid } \\
\text { vaccine }\end{array}$} & yes & $\%$ & 74.9 & 74.8 & 76.6 & 84.9 & 85.9 & 78.6 & $0.000^{* * *}$ \\
\hline & yes & CI & {$[71.9,77.8]$} & {$[71.9,77.5]$} & {$[73.2,79.6]$} & {$[81.6,87.8]$} & {$[82.1,89.0]$} & {$[77.1,80.0]$} & \\
\hline & Total & $\%$ & 100 & 100 & 100 & 100 & 100 & 100 & \\
\hline & $\mathrm{N}$ & & 1,235 & 1,148 & 1,006 & 880 & 713 & 4,982 & \\
\hline \multirow[t]{6}{*}{ taking iron } & no & $\%$ & 95.7 & 95.7 & 94.3 & 91 & 92.9 & 94.2 & \\
\hline & no & $\mathrm{CI}$ & {$[94.2,96.8]$} & {$[94.2,96.8]$} & {$[92.4,95.8]$} & {$[87.2,93.8]$} & {$[90.1,94.9]$} & {$[93.2,95.1]$} & \\
\hline & yes & $\%$ & 4.3 & 4.3 & 5.7 & 9 & 7.1 & 5.8 & $0.003^{* *}$ \\
\hline & yes & $\mathrm{CI}$ & {$[3.2,5.8]$} & {$[3.2,5.8]$} & {$[4.2,7.6]$} & {$[6.2,12.8]$} & {$[5.1,9.9]$} & {$[4.9,6.8]$} & \\
\hline & Total & $\%$ & 100 & 100 & 100 & 100 & 100 & 100 & \\
\hline & $\mathrm{N}$ & & 1,185 & 1,121 & 976 & 833 & 689 & 4,804 & \\
\hline RECODE of m15 & Home & $\%$ & 44.3 & 36.6 & 29.4 & 10.8 & 3.6 & 27.8 & \\
\hline \multirow[t]{5}{*}{ (wealth index) } & Home & CI & {$[40.7,48.0]$} & {$[32.7,40.6]$} & {$[26.0,33.1]$} & {$[8.4,13.7]$} & {$[2.2,5.8]$} & {$[25.8,29.9]$} & \\
\hline & $\begin{array}{l}\text { Health } \\
\text { Facility }\end{array}$ & $\%$ & 55.7 & 63.4 & 70.6 & 89.2 & 96.4 & 72.2 & $0.000^{* * *}$ \\
\hline & $\begin{array}{l}\text { Health } \\
\text { Facility }\end{array}$ & CI & {$[52.0,59.3]$} & {$[59.4,67.3]$} & {$[66.9,74.0]$} & {$[86.3,91.6]$} & {$[94.2,97.8]$} & {$[70.1,74.2]$} & \\
\hline & Total & $\%$ & 100 & 100 & 100 & 100 & 100 & 100 & \\
\hline & $\mathrm{N}$ & & 1,236 & 1,149 & 1,010 & 882 & 713 & 4,989 & \\
\hline
\end{tabular}

Regarding the timing of first ANC checkup, only $24 \%$ of the interviewed women made ANC visits within the first trimester. Out of these $30 \%$ representing the highest proportion were those in the highest wealth category compared to $26 \%$ for those in the lowest wealth category.

For receipt of tetanus taxoid vaccine, the results reveal that there was a significant difference between women in the highest and poorest wealth quintiles. The majority of women interviewed $(79 \%)$ received tetanus taxoid vaccine. Out of them, $86 \%$ belonged to the richest wealth category as opposed to the $75 \%$ who belonged to the poorest wealth category. 
The results in Table 1 also show that Iron table/syrup uptake was significantly low across all wealth quintiles (6\%). However, Iron intake increased with successively higher wealth categories (ranging from $4 \%$ for the poorest group to $7 \%$ for the richest group).

The results in Table 1 also reveal that the majority of the women which were interviewed delivered their newborns in a health facility ( $72 \%$ versus $28 \%)$ and that facility deliveries increased the wealth category higher. The results further reveal that almost all births among women in the richest wealth category $(96 \%)$ took place in a health facility as compared to only $56 \%$ of the women in the poorest wealth category.

Table 2 presents the adjusted odds ratios of the associations between the 5 indicators of maternal health and social, economic and demographic variables. Generally, the odds of a woman making 4 or more ANC visits and delivering in a health facility increased with successively higher wealth groups. According to the table, women who were in the middle, richer, and richest wealth quintiles had $30 \%, 50 \%$ and $180 \%$ respectively higher odds of making more than 4 ANC visits as compared to those in the poorest wealth quintile. The results also show that women in the richest wealth category had $120 \%$ higher odds of receiving their first ANC checkup within the first trimester of their pregnancies as compared to those in the poorest wealth category. Further, the women in the richer wealth category had $150 \%$ higher odds of taking Iron tablets/syrup as compared to those in the poorest category. In addition, women in the richest wealth quintile had significantly higher odds $(640 \%)$ of delivering their newborns in a health facility than those women in the richer, middle, and poorer wealth quintile $(180 \%, 50 \%$ and $30 \%$ ) respectively compared to those in the poorest quintile. 
Table 2. Adjusted regression results on the social, economic, and demographic factors associated with the individual indicators of maternal health for the most recent births

\begin{tabular}{|c|c|c|c|c|c|c|c|c|c|c|c|c|c|c|}
\hline \multicolumn{3}{|c|}{ anc4 } & \multicolumn{3}{|c|}{ anc3 } & \multicolumn{3}{|c|}{ tetanus_taxoid } & \multicolumn{3}{|c|}{ h42_ 1} & \multicolumn{3}{|c|}{ delivery_place } \\
\hline Odds & {$[95 \%$} & Inte & Odds & {$[95 \%$} & Inter & Odds & {$[95 \%$} & Inte & Odds & {$[95 \%$} & Inte & Odds & {$[95 \%$} & Inte \\
\hline Ratio & Conf. & rval] & Ratio & Conf. & val] & Ratio & Conf. & rval] & Ratio & Conf & rval] & Ratio & Conf. & rval] \\
\hline
\end{tabular}

\section{Wealth Index}

\begin{tabular}{|c|c|c|c|c|c|c|c|c|c|c|c|c|c|c|c|}
\hline 1. poorest & 1.0 & & & 1.0 & & & 1.0 & & & 1.0 & & & & & \\
\hline 2. poorer & 1.2 & 1.0 & 1.5 & 0.9 & 0.7 & 1.2 & 1.0 & 0.8 & 1.3 & 1.1 & 0.7 & 1.7 & $1.3^{* *}$ & 1.1 & \\
\hline 3. middle & $1.3^{*}$ & 1.1 & 1.6 & 1.0 & 0.7 & 1.3 & 1.0 & 0.8 & 1.3 & 1.5 & 1.0 & 2.4 & $1.5^{* * *}$ & 1.2 & \\
\hline 4. richer & $1.5^{* *}$ & 1.1 & 2.0 & 1.2 & 0.8 & 1.7 & 1.1 & 0.8 & 1.6 & $2.5^{*}$ & 1.2 & 5.0 & $2.8^{* * * *}$ & 1.9 & \\
\hline 5. richest & $2.8^{* * *}$ & 2.0 & 4.0 & $2.2^{* * *}$ & 1.5 & 3.3 & 1.0 & 0.7 & 1.6 & 1.8 & 0.9 & 3.8 & $7.4^{* * *}$ & 3.9 & \\
\hline
\end{tabular}

\section{Region}

1. central
2. copperbelt
3. eastern
4. luapula
5. lusaka
6. muchinga
7. northern
8. north western
9. southern
10. western

$\begin{array}{cccc}1.0 & 0.7 & 1.4 & 0.8 \\ 1.6^{* *} & 1.1 & 2.2 & 1.2 \\ 1.1 & 0.8 & 1.5 & 1.9 * * \\ 1.2 & 0.9 & 1.7 & 1.3 \\ 1.0 & & & 1.0 \\ 1.1 & 0.8 & 1.5 & 0.9 \\ 1.0 & 0.7 & 1.5 & 0.9 \\ 1.1 & 0.8 & 1.5 & 0.9 \\ 1.2 & 0.9 & 1.8 & 1.0 \\ 1.0 & 0.7 & 1.4 & 0.8\end{array}$

\begin{tabular}{lll}
0.6 & 1.2 & $0.4^{* * *}$ \\
0.9 & 1.7 & $0.4^{* * *}$ \\
1.3 & 2.7 & $0.5^{* *}$ \\
0.9 & 2.1 & $0.6^{*}$ \\
& & \multicolumn{2}{c}{1.0} \\
0.6 & 1.4 & $0.5^{* *}$ \\
0.6 & 1.3 & $0.5^{*}$ \\
0.6 & 1.3 & $0.4^{* * *}$ \\
0.7 & 1.5 & $0.4^{* * *}$ \\
0.5 & 1.2 & $0.4^{* * *}$
\end{tabular}

0.2
0.3
0.3
0.4
0.3
0.3
0.2
0.3
0.3

\begin{tabular}{cccccr}
0.6 & $0.3^{* *}$ & 0.1 & 0.7 & $0.2^{* * *}$ \\
0.7 & 1.3 & 0.8 & 2.3 & 0.6 \\
0.8 & 1.2 & 0.6 & 2.2 & 1.2 \\
1.0 & 0.6 & 0.3 & 1.2 & & 0.9 \\
& 1.0 & & & & 1.0 \\
0.7 & 0.9 & 0.4 & 1.8 & \multicolumn{2}{l}{0.7} \\
0.9 & 0.9 & 0.4 & 1.7 & $0.4^{* *}$ & \\
0.6 & $0.4^{*}$ & 0.2 & 0.9 & \multicolumn{2}{c}{1.0} \\
0.6 & 0.7 & 0.3 & 1.6 & $0.4^{* * *}$ \\
0.7 & 1.6 & 0.8 & 3.1 & $0.5^{*}$ &
\end{tabular}

$\begin{array}{ll}0.1 & 0.4 \\ 0.3 & 1.0 \\ 0.7 & 2.1 \\ 0.5 & 1.6 \\ & \\ 0.4 & 1.3 \\ 0.2 & 0.7 \\ 0.6 & 1.9 \\ 0.2 & 0.6 \\ 0.3 & 1.0\end{array}$




\section{Open Science Journal}

Type of Place of

Residence

1. urban

1.0

2. rural

$1.5 * * *$

$1.2 \quad 1.9 \quad 1.5 * *$

1.2

$2.0 \quad 0.6^{* * *}$

.0

$\begin{array}{lll} & & 1.0 \\ 0.4 & 0.7 & 1.4\end{array}$

0.8

$2.40 .4^{* * *}{ }^{1.0}$

$0.3 \quad 0.6$

Literacy

not literate

literate

1.0

$\begin{array}{llll}1.0 & 0.9 & 1.1 & 0.9\end{array}$

0.7

1.0

$\begin{array}{lll}0.0 & 1.0\end{array}$

0.6

$1.21 .5^{* * *}$

$1.3 \quad 1.8$

Mothers age at Birth

$<20$

1.0
1.0

20-34

1.0

$0.8 \quad 1.2$

1.0
0.9

0.8

$\begin{array}{ll} & 1.0 \\ 1.2 & 0.9\end{array}$

$\begin{array}{lll} & & 1.0 \\ 0.7 & 1.2 & 1.0\end{array}$

0.7

\begin{tabular}{ll} 
& \multicolumn{2}{c}{1.0} \\
1.6 & $0.6^{* * *}$ \\
1.4 & $0.5^{* * *}$
\end{tabular}

$\begin{array}{ll}0.5 & 0.8\end{array}$

$35+$

$\begin{array}{lll}1.1 & 0.9 & 1.5\end{array}$

0.8

0.2

0.4

0.8

0.5

Marital Status

married

1.0

$\begin{array}{ll}0.8 & 1.0\end{array}$

1.0

1.0

$\begin{array}{ll}1.0 & 1.0\end{array}$

$\begin{array}{llll}1.0 & 0.8 & 1.2 & 1.2\end{array}$

$\begin{array}{ll}0.9 & 1.7\end{array}$

0.9

$0.6 \quad 1.2$

0.8

0.5

1.4

0.8

0.6

1.1

Media Exposure

No access/exposure

$\begin{array}{lll}1.0 & 1.0 & 1.0\end{array}$

Access to

$0.8 \quad 1.1$

0.9

0.8

1.1

0.9

$\begin{array}{lll}0.8 & 1.1 & 1.2\end{array}$

1.0

$\begin{array}{lll}0.8 & 1.6 & 1.2^{*}\end{array}$

1.0

media/exposed 
For region, Table 2 reveals that women on the Copperbelt province had $60 \%$ higher odds of making more than 4 ANC visits as compared to those women in Lusaka. Furthermore, women in Eastern province had $90 \%$ higher odds of making the first ANC visit within three months of their pregnancies as compared to those in Lusaka province. The results also reveal that the odds of the mother receiving Tetanus Taxoid vaccination varied across provinces; varying from $40 \%$ to $60 \%$ lower than the odds for the mothers in Lusaka province. The odds of taking iron tablets/syrup among mothers in Central and North-Western provinces were $70 \%$ and $60 \%$ respectively. This, however, is lower than those mothers in Lusaka province. Consequently, mothers in Central, northern, southern, and western region had $80 \%, 60 \%, 60 \%$ and $50 \%$ respectively lower odds of delivering their newborns in a health facility as compared to those mothers in Lusaka province.

The results also reveal that literate women have $50 \%$ higher odds of delivering their newborns in a health facility as compared to those who are not literate.

For type of place of residence, the results in Table 2 reveal that mothers in rural areas had $50 \%$ higher odds of making 4 or more ANC visits and receiving ANC within the first 3 months of their pregnancies as compared to women in urban areas. On the other hand, mothers in rural areas had lower odds of uptake of tetanus taxoid vaccination and delivering their newborns in a health facility $40 \%$ and $60 \%$ respectively as compared to those in the urban areas.

The results also show that women in the age group $35+$ had lower odds of uptake of the tetanus taxoid vaccine and delivering their newborns in a health facility $70 \%$ and $50 \%$ respectively as compared to those in the $<20$ age category. Women in the age group 20-34 had 40\% lower odds of having a health facility birth compared to those in the $<20$ age category. In addition, mothers who had media access had $20 \%$ higher odds of delivering their newborns in a health facility compared to those who had no access or exposure to media.

Table 3 shows the adjusted regression results on the social, economic, and demographic factors associated with a composite measure of maternal health for the most recent birth. The results reveal that women in the middle and richer wealth quintiles have higher odds of experiencing good maternal health outcomes as compared to those in the poorest wealth quintile $(80 \%, 180 \%)$ respectively. Also, the odds are considerably very high for the women in the richest wealth category $(1420 \%)$ as compared to the women in the poorest wealth category. The results also reveal that women in Central, Copperbelt, Northern, North-Western, Southern, and Western provinces had lower maternal outcomes as compared to those from Lusaka province. According to the results, their odds ranged from $70 \%$ to $80 \%$ lower than the women from Lusaka province. Further, the results reveal that literate women had $50 \%$ higher odds of having good maternal health outcomes as compared to those who were not literate. 
Table 3. Adjusted regression results on the social, economic, and demographic factors associated with a composite measure of maternal health for the most recent births

maternal_health $\quad$ Odds Ratio $\quad[95 \%$ Conf. $\quad$ Interval

v190

1. poorest

1.0

2. poorer

1.2

3. middle

$1.8^{* *}$

4. richer

$2.8^{*}$

5. richest

v024

1. central

2. copperbelt

3. eastern

4. luapula

5. Lusaka

6. muchinga

7. northern

8. north western

9. southern

10. western

v025

1. urban

2. rural

literacy _ 1

not literate

Literate

0.5

1.0

0.4

$0.3^{*}$
$0.3^{*}$
$0.2^{*}$
$0.2^{*}$

$0.3^{*}$

$.3^{*}$

$0.2^{*}$

0.8

1.0

age_birth

$<20$

20-34

$35+$

$0.3^{* * *}$
0.0

0.5

0.1

0.9

0.2

1.9

0.1

1.7

0.1

1.4

0.1

0.9

0.1

1.0

0.1

0.8

0.1

marital_status

never married

Married

1.0

1.0

0.6

media_exposure

No access/exposure

1.0

0.9

0.6 


\section{Discussion}

The study examined the impact of wealth status and other social, economic and demographic factors on maternal health in Zambia using the most recent Zambia Demographic and Health Survey (ZDHS) 2013-14. The results reveal that slightly over half of the women interviewed made more than 4 ANC visits, and only about a quarter had their first ANC visit within the first trimester of their pregnancies. When compared to a study conducted by [24], the results show that there has been some improvement in the two indicators despite the timing of first ANC within the first trimester being low (24\%). In the study by [24], it was found that a considerable proportion of parturient women in Zambia continued to receive fewer than the minimum number of required visits (four) and/or did not receive antenatal check-ups in the first trimester, even after the adoption of the Focused Antenatal Care (FANC). The improvement could be explained by the fact that over the years, the Government of the Republic of Zambia with its cooperating partners has initiated several programs to improve maternal health such as the Saving Mothers Giving Life intervention in collaboration with the United States Government initiated in 2011 whose aim was to reduce maternal mortality by $50 \%$ in just one year [12]. The study has also revealed that the number of ANC visits increased as the women's wealth increased. This result is in line with other studies that have found wealth to influence the use of health services positively $[15,25]$. The study has also demonstrated that receipt of tetanus taxoid vaccination and uptake of iron tablets/syrup increased as the wealth index increased. However, while the overall proportion of taking tetanus taxoid vaccination was high, the proportion for iron uptake was rather low. This finding is paradoxical due to the fact that both indictors are part of the antenatal care services provided to women when they attend antenatal care. Therefore, it is expected that when the proportion of one is high, then the proportion of the other will also follow suit. The argument that can be forwarded for this adverse iron uptake result is that, women may perceive the period for taking the iron tablets/syrup to be too long and as such shun taking it. The Ministry of Health [2] advises women to take an iron tablet daily throughout their pregnancy and lactating period starting from the second trimester and continuing to 45 days after childbirth. The results further show that about $72 \%$ of women gave birth in a health facility of which the majority were from the highest wealth quintile. This result is in conformity with a study by [16]. It follows that giving birth in a health facility reduces maternal deaths arising from complications of pregnancy. Subsequently, mostly wealthy women are more likely to deliver in health facilities because they have the capacity to pay for health care.

The regression results for the factors associated with individual indicators of maternal health reveal the following: The factors associated with the number of ANC visits include wealth, region, and type of residence. In terms of wealth, the results show that the odds of the mother making 4 or more ANC visits increased as the wealth increased. This result is consistent with the findings of a study by [20] in which it was found that the number of ANC visits increased as the wealth index increased. This finding can be explained by the fact that wealthier women have a higher ability to pay and therefore seek more ANC as compared to those who are poor. In terms of region, only women on the Copperbelt province had 
higher odds of making 4 or more ANC visits as compared to Lusaka province. Thus, this can be explained by the fact that being a Mining province, a lot of health infrastructure has been built by the mines and as such, mothers have access to maternal health services. In terms of type of place of residence, the results reveal that women in rural areas had 50\% higher odds of making 4 or more ANC visits compared to women in urban areas. This result is contrary to expectation. However, it could be explained by the initiatives being undertaken by the government which are mainly targeted at rural areas such as access to ANC via free public provision [24]. It is also performed by the Saving Mother Giving Life intervention initiated in 2011 [12].

The factors associated with the timing of ANC visits include wealth, region, and type of residence. In terms of wealth, results show that the odds of the mother making her first ANC visit within the first trimester of her pregnancy was higher for the women in the richest wealth category as compared to those in the poorest wealth category. This result is consistent with the result by [26] in a study conducted in Pakistan, in which he found substantial variation in the timing of the first ANC check-up by household wealth. According to [26], the median time of the first ANC check-up was 3 months for women in the richest wealth quintiles, and 7 months for women in the poorest wealth quintiles. The result from this study is in line with the [23] recommendation that in order to detect and effectively treat underlying problems, the first antenatal care visit should occur as early as possible, and preferably within the first trimester. This result could be explained by the fact that wealthier women stand to be more knowledgeable about the importance of ANC visits during the early stages of pregnancy and as such will tend to make their first ANC visit within the first trimester of their pregnancy. In terms of region, women in Eastern province had $90 \%$ higher odds of making their first ANC visit within the first trimester of their pregnancy as compared to Lusaka province. Therefore, this could be attributed to the initiatives by the government and its cooperating partners such as the Saving Mother Giving Lives initiative that was rolled out in two districts of Eastern province (Nyimba and Lundazi) in 2011 [12]. In terms of type of place of residence, the results reveal that women in rural areas had $50 \%$ higher odds of making their first ANC visit within the first trimester of their pregnancy as compared to those in urban area. Thus, the reasons could also be as a result of the pro-ANC initiatives by the government with its cooperating partners.

The factors associated with the uptake of tetanus taxoid vaccination included region, type of place of residence, and mother's age at birth. The results reveal that the odds of the mother receiving tetanus taxoid vaccination varied across provinces and generally the odds for all provinces were lower than Lusaka province which was the reference category. This result could be explained by the choice of reference category which happens to be the country's capital. In addition, it has the majority of health facilities and therefore access and utilization of ANC services is not a problem. Low access and utilization of ANC in the other provinces, however, leads to the lack of knowledge of the importance and need for tetanus taxoid vaccination. Hence, this explains the low odd of tetanus taxoid vaccination results in these provinces. The mothers in the age group $35+$ had $70 \%$ lower odds of receiving tetanus taxoid vaccination than the mothers in $<20$ age group. This result could be explained by the fact that women $30+$ once they have gone through the experience of child birth without any 
complications in the past, they may be reluctant to take tetanus taxoid vaccination as they may feel it has no importance.

The factors associated with the uptake of Iron tablet/syrup included wealth and region. The results show that women in the richer wealth category had $150 \%$ higher odds of taking Iron tablets/syrup as compared to those in the poorest category. This shows that wealth is a significant factor in explaining variations in Iron intake in the sense that women who are wealthy are able to afford the purchase of Iron tablets/syrup. In terms of region, the odds of taking Iron tablets/syrup among mothers in Central and North-western provinces were lower than those mothers in Lusaka province. This could be attributed to the fact that these two provinces, predominantly rural and health facilities, are very far apart. As a result, access to Iron tablets/ syrup may be a problem.

The factors associated with place of delivery included wealth, region, type of place of residence, literacy, and mothers age at birth. Generally, the odds of a woman delivering in a health facility increased with successively higher wealth groups. [16] showed that household wealth is positively and significantly associated with choosing health facility for delivery. According to the table, women who were in the Poorer, middle, richer and richest wealth quintiles had $30 \%, 50 \%, 180 \%$ and $640 \%$ respectively higher odds of delivering their newborns in a health facility compared to those in the poorest quintile. This result is in line with the finding of [20] in their study of the Wealth Index and Maternal Health Care. The explanation for this is that with higher wealth, the mother is able to have access to health facilities where she can receive proper maternal health care services during delivery from well-trained medical personnel. The results also reveal that mothers in Central, Northern, Southern, and Western provinces had lower odds of delivering their newborns in a health facility as compared to those mothers in Lusaka province. In addition, mothers in rural areas also had lower odds of delivering their newborns in a health facility as compared to those in the urban areas. This finding is consistent with the results of [18] who found that women residing in rural areas were less likely to deliver at healthcare facilities than their urban counterparts. This finding could be explained by the fact that these provinces are predominantly rural and as such, the health facilities could be few in terms of numbers thereby making access as a result of distance a serious problem for the mothers who want to deliver their newborns. The other reason that could explain these lower odds is the poor quality of health care services being provided in the Health Centre's in these areas; for instance, lack of medical personnel, long queues before being attended to, and lack of medicines, lack of ambulance or emergency transfer services coupled with poor road infrastructure to name but a few. Poor quality of health services may result in the mother's loss of confidence in the health care system and therefore reduces their health seeking behavior.

The results also reveal that literate women have $50 \%$ higher odds of delivering their newborns in a health facility as compared to those who are not literate. This finding is consistent with a similar finding in a study by [27] who found that, when the influences of other intervening factors were controlled, education emerged as the single most important determinant of maternal health care utilization in India. This finding can be explained by the fact that, with education, women are given awareness of the need to utilize health care services where they can be attended to by a qualified health practitioner during delivery 
so as to avoid birth related complications which may result to death. The results also show that as women's age increased, their odds of delivering in a health facility declined as compared to those in the youngest age category. This could be explained by the fact that younger women might have an enhanced knowledge of available health care services and place more value upon modern medicine as a result of the improvement in educational opportunities for women in recent years. In addition, mothers who had media access had higher odds of delivering their newborns in a health facility compared to those who had no access or exposure to media. The explanation to this is that access to media enhances women's knowledge about ANC and as such, they tend to know the benefits and importance of delivering their newborns in a health facility.

From the computed composite measure for maternal health, only wealth, region, literacy, and age of the mother were found to be significant in explaining the variations in maternal health. The results reveal that after controlling the effect of other variables in the model, maternal health improved as the wealth index increased. Thus, this implies that the poor are excluded from benefiting of maternal health services. This result also just reaffirms the importance of wealth in improving maternal health outcomes in Zambia in the sense that with increased wealth, the ability to pay the costs (direct and indirect) associated with utilization of maternal health services increases. The results for region also just reaffirm the findings in this study that apart from Lusaka and the Copperbelt provinces, the remaining provinces are predominantly rural. As a result, distance and quality of health services remain major bottlenecks in accessing good maternal health care by mothers. Mothers who were literate and $35+$ were positively associated with good maternal health outcomes. With education, a mother has knowledge of the importance of seeking maternal health care services. She is also aware that maternal health care services are provided without charge in government health facilities and will therefore seek these services. According to the Grossman model, age increases the rate of depreciation of the health of the individual. It may therefore be possible that, the older women may patronize health services more than the younger ones [28]. Therefore as a mother advances in her age, she tends to seek more maternal health services as she may have knowledge of potential complexities that arises before, during, and after child birth.

\section{Conclusion}

Generally, the wealth status of the mother and region of residence were found to be a significant and very important factor associated with maternal health for all the 5 indicators independently and for the composite measure of maternal health in the study. It can therefore be inferred from the results that women in higher wealth quintiles are more likely to make more ANC visits than women in the lowest wealth quintile. Furthermore, the timing of the first ANC visit is higher among the women in the higher wealth quintiles. In addition, there was a positive association between wealth and the other three maternal health indicators (receipt of tetanus taxoid vaccination, uptake of Iron table/syrup, and delivery in a health facility). From these results, it can be deduced that even 
though maternal health services provided by the government in Zambia are free, there are usually some direct or indirect cost attached to them thereby making access for those in lower wealth categories unattainable.

Other factors such as type of place of residence were only associated with mothers making 4 or more ANC visits, receiving ANC within the first 3 months of pregnancy, uptake of tetanus taxoid vaccination and mothers delivering their newborns in a health facility, and also the composite measure of maternal health. These results reveal that apart from Lusaka and the Copperbelt provinces, most parts of Zambia are predominantly rural and therefore distance and quality of health care remains a major challenge to accessing good maternal health care by mothers.

Literacy was only significant for uptake of Iron tablet/syrup, but is also highly significant for the composite measure of maternal health. The significant impact of education in improving maternal health outcomes is evidence that educated women have better knowledge and information on modern medical treatment. Also, they are aware that maternal health care services are provided without charge in government health facilities and will therefore seek these services.

The results also show that women in the older age categories had lower odds of uptake of the tetanus taxoid vaccine and delivering their newborns in a health facility as compared to the younger women. This result shows that as a mother advances in her age, she tends to seek more maternal health services as she may have knowledge of potential complexities that arise before, during, and after child birth. In addition, the results show that access to media enhances women's knowledge about ANC care and as such, they tend to know the benefits and importance of delivering their newborns in a health facility.

Lastly, the study also reveals that despite the low proportion of first ANC visit being within the first trimester of pregnancy, there has been an increase in the frequency and timing of ANC visits among women in Zambia.

\section{Policy Implications}

Since wealth has been found to be a very important factor associated with maternal health care in Zambia, there is need for policy to be formulated that provides support to women within the lowest wealth quintiles so as to enable them access maternal health services. Policy could be in the form of ensuring that essential drugs such as Iron tablets/syrup and tetanus taxoid vaccines are at least available at the health center to relieve the mother of the costs they may have to incur in purchasing the medicine. Policy could also be aimed at ensuring the availability of a number of medical health personnel so that the mothers do not spend too much time at the health centre which may be a discouragement in the use of the service. Policy should also focus on encouraging women to pursue education and increase information dissemination on the free provision of maternal healthcare services to women in the hard-to-reach remote areas. Policy makers may also endeavor to improve the distribution of health facilities in rural areas so as to mitigate the challenges of accessibility as a result of distance. There is also need to implement sensitization programs targeting campaigns that can capture young mothers on the importance of maternal health services so as to increase their utilization of the services. 


\section{Study Limitations}

This study had three major limitations: First, due to the cross-sectional nature of the survey, it cannot offer any insights into cause-and-effect relationships between the independent and dependent variables in this study. Secondly, the study was only restricted to the factors collected in the ZDHS, other factors such as distance to health facility could not be examined as it was not collected in Demographic Health Surveys. Lastly, the missing and unknown values in the dataset were dropped from the analysis. However, this may result in biased and inefficient statistical estimates due to the loss of information.

\section{References}

1. WHO, UNICEF, UNFPA, World Bank Group and the United Nations Population Division. Trends in Maternal Mortality: 1990 to 2015 Estimates by WHO, UNICEF, UNFPA, World Bank Group and the United Nations Population Division, WHO Press, Geneva, 2015.

2. Ministry of Health (Zambia). The Zambia 2008 Countdown to 2015 Conference Report. Held 20th - 21st August 2008 at The Mulungushi International Conference Centre, MOH, Lusaka, 2008.

3. Babalola S., and A. Fatusi. Determinants of use of Maternal Health Services in Nigeria Looking Beyond Individual and Household Factors, BMC Pregnancy and Childbirth. 9:43, 2009, doi: 10.1186/1471-2393-9-43

4. Birmeta K., Dibaba Y. and D. Woldeyohannes. Determinants of Maternal Health Care Utilization in Holeta Town, Central Ethiopia, BMC Health Services Research.13:256, 2013, doi: 10.1186/1472-6963-13-256.

5. Sialubanje C., Massar K, Hamer D. H. and R. A. C. Ruiter. Understanding the Psychosocial and Environmental Factors and Barriers Affecting Utilization of Maternal Healthcare Services in Kalomo, Zambia: A Qualitative Study, Health Education Research, 29:3, pp. 521-532, 2014.

6. WHO, World Health Organization. World Health Statistics: Monitoring Health for the SDGs. WHO press, Geneva, 2016.

7. World Health Organization. Maternal Mortality, 2015 Fact Sheet No.348. http://www.who.int/mediacentre/factsheets/fs348/en/. Accessed on September 15, 2016.

8. World Health Organization. Maternal Mortality. http://www.who.int/topics/maternal_health/en/. Accessed on September 16, 2016.

9. Hunt P. H. and J. B de Mesquita. Reducing Maternal Mortality: The Contribution of the Right to the Highest Attainable Standard of Health Project Report. Human Rights Centre, Colchester, Essex, 2014.

10. Central Statistical Office (CSO) [Zambia], Ministry of Health (MoH) [Zambia], and ICF International. Zambia Demographic and Health Survey 2013-14. Rockville, Maryland, Central Statistical Office, Ministry of Health, and ICF International, 2014.

11. Geloo Z. Diverse Factors Linked to Maternal Deaths in Zambia, Population Reference Bureau, 2003.

12. United States Government Zambia Interagency Team. Global Health Initiative Strategy Zambia 2011 -2015, 2012.

13. United Nations Development Programme (UNDP). Millennium Development Goals Progress Report 2013, United Nations, New York, 2013

14. Central Statistics Office (CSO). 2010 Census of Population and Housing, CSO, Lusaka, 2012.

15. Arthur E. Wealth and Antenatal Care Use: Implications for Maternal Health Care Utilisation in Ghana. Health Economics Review, 2:14, 2012, doi:10.1186/2191-1991-2-14.

16. Celik Y. and D.R. Hotchkiss. The Socio-economic Determinants of Maternal Health Care Utilization in Turkey. Social Science Medical, 50:12, pp. 1797-1806, 2000, doi: org/10.1016/S0277-9536(99)00418-9 
17. Chakraborty N., M. A. Islam, R. I. Chowdhury, W. Bari and H. H. Akhter. Determinants of the Use of Maternal Health Services in Rural Bangladesh. Health Promotion International. 18:4, pp. 327-337, 2003, doi: 10.1093/heapro/dag414.

18. Mazambani D., L. Chigusiwa, V. Mudavanhu, S. Bindu and L. Muchabaiwa. Determinants of Maternal Healthcare Utilization in Zimbabwe. International Journal of Economic Sciences and Applied Research, 5:2, Pp. 145-162, 2012.

19. Chama C. M. C. An Economic Analysis of Maternal Health Care in Zambia. A dissertation submitted in partial fulfilment of the requirement for the Degree of Doctor of Philosophy. University of Pretoria, 2013.

20. Goel M. K., P. Roy, S. K. Rasania, S. Roy, Y. Kumar and A. Kumar. Wealth Index and Maternal Health Care: Revisiting NFHS-3. Indian Journal of Public Health, 59:3, pp. 217-219, 2015, doi:10.4103/0019-557X.164665.

21. Khanna R. and B. Subha Sri. Common Health India. It is adapted from a chapter in a forthcoming volume: Nambiar D. and A. Muralidharan (Eds.) The Art of the Possible: Understanding and Acting on the Social Determinants of Health in India. Forthcoming with Orient Blackswan. http://www.who.int/maternal_child_adolescent. Accessed on 20 September, 2016.

22. Wang W., G. Temsah and L. Mallick. Health Insurance Coverage and Its Impact on Maternal Health Care Utilization in Low- and Middle-Income Countries. DHS Analytical Studies 45. Rockville, Maryland, ICF International, 2014.

23. World Health Organization (WHO). Standards for Maternal and Newborn Health: Provision of Effective Antenatal Care. Geneva, WHO, 2004. Available online at http://whqlibdoc.who.int/hq/2007/a91272.pdf.

24. Chiliba C. M. C. and S. F. Koch. Utilization of Focused Antenatal Care in Zambia: Examining Individual and Community-Level Factors Using a Multilevel Analysis. Health Policy and Planning, 1-10, 2013, doi:10.1093/heapol/czt099

25. Ortiz A. V. Determinants of demand for antenatal care in Colombia. Health Policy, 86, 363372, 2007, .doi.org/10.1016/j.healthpol.2007.12.002

26. Tappis H. and S. Agha. The Timing of Antenatal Care: Implications for Equity in the Provision of Quality Care Presentation at the Global Maternal Newborn Health Conference, October 21, 2015

27. Srivastava A., Mahmood S., Mishra P, V. Shrotriya. Correlates of Maternal Health Care Utilization in Rohilkhand Region, India. Annals of Medical and Health Sciences Research. 4:3, pp. 417-425, 2014, doi:10.4103/2141-9248.133471.

28. Elo I. T. Utilization of Maternal Health-Care Services in Peru: The Role of Women's Education. Health Transit Review. 2:1, pp. 49-69, 1992. 